\title{
Evaluation of a new Rapid Antimicrobial Susceptibility system for Gram-negative and Gram-positive bloodstream infections: speed and accuracy of Alfred 60AST
}

\author{
Vanesa Anton-Vazquez ${ }^{1,3^{*}}$ (D), Samuel Adjepong ${ }^{2}$, Cristina Suarez ${ }^{1}$ and Timothy Planche $e^{1,2,3}$
}

\begin{abstract}
Background: Blood stream infections (BSIs) are a major cause of morbidity and mortality. The time from taking blood cultures to obtain results of antibiotic sensitivity can be up to five days which impacts patient care. The Alfred $60 \mathrm{AST}^{\mathrm{TM}}$ can reduce laboratory time from positive culture bottle to susceptibility results from 16 to $25 \mathrm{~h}$ to 5-6 h, transforming patient care. To evaluate the diagnostic accuracy of a rapid antimicrobial susceptibility system, the Alfred $60 \mathrm{AST}^{\mathrm{T}}$, in clinical isolates from patients with BSIs and confirm time to results. $301 \mathrm{Gram}$-negative and 86 Gram-positive isolates were analysed directly from positive blood culture bottles following Gram staining. Antimicrobial susceptibility results and time-to-results obtained by rapid Alfred 60 AST system and BD Phoenix were compared.
\end{abstract}

Results: A total of 2196 antimicrobial susceptibility test results (AST) were performed: 1863 Gram-negative and 333 Gram-positive. AST categorical agreement (CA) for Alfred 60 ASTTM was 95\% (1772/1863) for Gram-negative and 89\% (295/333) for Gram-positive isolates. Gram-negative CA: ampicillin 96\% (290/301); ciprofloxacin 95\% (283/297); ceftriaxone 96\% (75/78); meropenem 97\% (288/297); piperacillin-tazobactam 95\% (280/295); gentamicin 94\% (279/ 297) and amikacin 93\% (277/298). The median time to susceptibility results from blood culture flagging positive was $6.3 \mathrm{~h}$ vs $20 \mathrm{~h}(p<0.01)$ for Alfred system vs BD Phoenix ${ }^{\mathrm{TM}}$.

Conclusion: Alfred 60 AST system greatly reduced time to antimicrobial susceptibility results in Gram-negative and Gram-positive BSIs with good performance and cost, particularly for Gram-negative bacteraemia.

Keywords: Rapid diagnostics. Bloodstream infection. Bacteraemia. Antimicrobial susceptibility testing. Gramnegative bacteria. Gram-positive bacteria

\section{Background}

Blood stream infections (BSIs) are worldwide, a major cause of morbidity and mortality [1]. The clinical laboratory plays a key role in the diagnosis of BSIs and provides antimicrobial susceptibility results which are crucial in clinical decision-making. Blood cultures remain the main way to identify blood stream infection. Despite optimisation in laboratory workflows that have

\footnotetext{
* Correspondence: vanesa.anton.v@gmail.com

'St. George's University of London. Institute for Infection and Immunity, London SW17 ORE, UK

${ }^{3}$ Infection Care Group, St George's University Hospitals NHS Foundation Trust, Blackshaw Road, London SW17 OQT, UK

Full list of author information is available at the end of the article
}

decreased time-to-result (Anderson, [2]), the time from taking a blood culture to obtaining a result of antimicrobial susceptibilities can be up to 5 days [3]. The time to positivity, time from blood culture collection to bottle flagging positive, is typically 12 to $24 \mathrm{~h}$ [1]. The subsequent mean time to identify bacterial species and obtain antimicrobial susceptibilities, using standard methods, is a further $36 \mathrm{~h}[4]$.

Phenotypic antimicrobial susceptibility tests (AST) generally relies on detecting bacterial growth in the presence of antibiotic. Standard methods of testing used in routine diagnostic laboratories for patient care include broth or agar micro-dilution, disc diffusion or antibiotic

(c) The Author(s). 2019 Open Access This article is distributed under the terms of the Creative Commons Attribution 4.0 International License (http://creativecommons.org/licenses/by/4.0/), which permits unrestricted use, distribution, and 
gradient strips, which require around $24-72 \mathrm{~h}$ to complete [5]. A number of automated systems for testing antimicrobial susceptibility are increasingly used such as BD Phoenix ${ }^{\mathrm{mi}}$ (Becton Dickinson, USA), VITEK$2^{\mathrm{rm}}$ (BioMerieux, France) and MicroScan ${ }^{\mathrm{Tm}}$ (Beckman, USA) which have a time to results ranging between 12 and $24 \mathrm{~h}$ after positive culture [6].

The time to clinically useful antimicrobial susceptibility data has an impact on patient care either to change to an effective antibiotic in the case of a resistant isolates or to focus to a narrower spectrum antibiotic for susceptible isolate. The development of rapid diagnostics is a key aim in the control of the rise in antibiotic resistance [7]. The use of genotypic methods such as nucleic acid amplification tests to detect antibiotic resistance in routine blood cultures has not been widely adopted due to high cost [8], lack of sensitivity when using blood direct from the patient [9] and inability to detect many resistance mechanisms and hence "rule-in" the use of antibiotics when no resistance marker is detected [10]. This highlights the need to increase the speed of phenotypic methods to shorten the time to obtain antimicrobial susceptibility results [11].

The Alfed $60 \mathrm{AST}^{\mathrm{Tm}}$ (Alifax, Italy) is a CE marked automated commercial laser-scattering based in-vitro diagnostic (IVD) providing antimicrobial susceptibility results directly from positive blood culture bottles within 4-6 h. The Alfred 60 AST $^{\mathrm{mon}}$ uses light scatter to detect bacterial growth in a liquid culture broth. In brief, $30 \mu \mathrm{L}$ of blood from a positive blood culture is inoculated into a broth that is incubated, then light scatter is measured until it reaches a turbidity of 0.4-0.6 McFarland. A series of tubes containing antibiotics are then robotically inoculated and bacterial growth measured by light scatter providing real time bacterial growth curves. Growth curves of bacteria over $3-5 \mathrm{~h}$ in the tested antibiotics are compared to growth a positive control (no antibiotic) and percentage of inhibition of growth (\%PIC) is calculated. The \%PIC is compared between control and antibiotic tubes and reported as resistant, intermediate and sensitive categories, according to the range of inhibition (Sensitive result: Inhibition of growth $>65 \%$; Intermediate: Inhibition of growth between 65 and 50\%; Resistant: Inhibition of growth $<50 \%$ ). To grant a reliable detection of the growth inhibition (keeping as reference the "Reference Vial") a proper growth must be detected ( $\geq$ $700.00 \mathrm{CFU} / \mathrm{ml}$ ), if not the test is not considered valid.

The system is based on a light scattering technique, A light laser beam which is oriented trough a sample and two photo detectors placed at $30^{\circ}$ and $90^{\circ}$ receive signals generated by the light scattered by the bacteria present in the vial. Growth kinetic curves are obtained for each detector and displayed in two curves. Double reading channel (2 detectors) is important for samples with high initial turbidity level. If 1 detector (the 1st one) becomes saturated due to the high sample's turbidity then the 2nd detector is able to detect signals. Finally, a mathematical algorithm selects the best reading detector channel for the calculation of the inhibition of growth. (Fig. 1).

The panel of antibiotics tested can be predefined by users. Hands on time for each run is about $15 \mathrm{~min}$. In addition, the system requires a daily set up of approximately $10 \mathrm{~min}$ and a weekly maintenance wash out of $30 \mathrm{~min}$. Up to 60 individual tests can be performed in a run, the number of isolates tested depends on the number of antibiotics tested (for example 10 isolates may be tested for 6 antibiotics each).

The time to result is unaffected by the number of antibiotics or samples tested. Alfred 60 AST recognizes each reference vial followed by its respective antibiotic vials as a single assay and has the capacity to initiate multiple single susceptibility assays at the same time for different isolates.

The purpose of this study was to compare the accuracy and speed of results of the Alfred $60 \mathrm{AST}^{\mathrm{mm}}$ with the BD Phoenix ${ }^{\text {Tw }}$ (Becton Dickinson, USA) automated susceptibility testing system, a broth-based microdilution test that utilizes a redox indicator to enhance the detection of organism growth, which is the standard of care in our routine diagnostic laboratory.

\section{Results}

Antimicrobial susceptibility results of Alfred 60 system

Antimicrobial susceptibility results were performed for 387 clinical positive blood culture bottles that were monomicrobial on Gram stain (301 Gram-negative and 86 Gram-positive) with a total of 2196 individual antimicrobial test results (1863 Gram-negative and 333 Gram-positive). Eighteen samples (5\%) were excluded from the analysis. Excluded samples comprised 3 polymicrobial samples that were not detected during Gram stain and 15 non valid results included 10 system technical failure before AST performance and 5 failures of no bacterial growth in the reference vial on Alfred 60 AST system, including: 1 Acinetobacter baumanni; 1 Achromobacter spp; 1 Bacteroides fragilis; 1 Pseudomonas aeruginosa; 1 Klebsiella oxytoca.

$71 \%(213 / 301)$ of the Gram-negative and 43\% (37/86) of the Gram-positive isolates were resistant to at least one antibiotic. 9\% (28/301) of the Gram-negative organisms were identified as Extended-spectrum betalactamases (ESBLs) as defined by BD Phoenix ${ }^{\mathrm{Tm}}$ expert rules for resistance. Fig. 3.

There were a total of 301 valid Alfred 60 AST results for Gram-negative monomicrobial isolates. Data were available on the following organisms: 209 E.coli; 57 Klebsiella spp; $10 \mathrm{P}$. aeruginosa; 12 Proteus spp; 7 


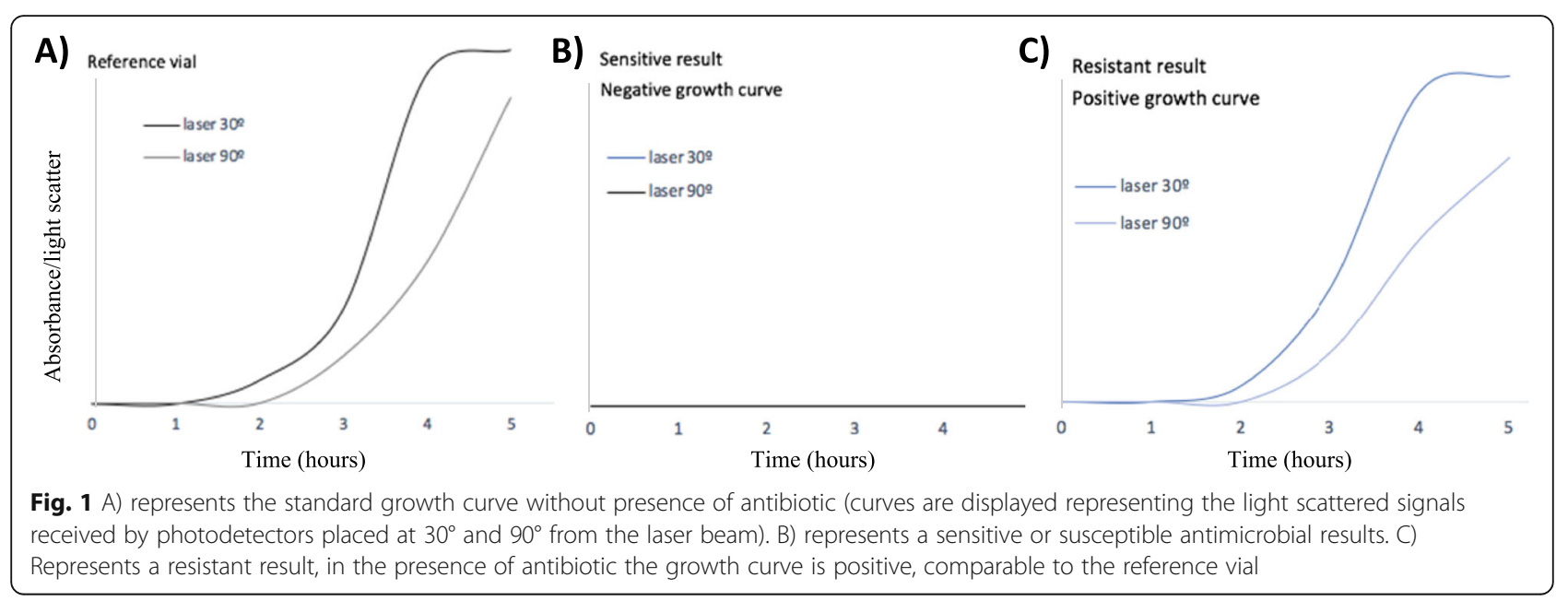

Enterobacter spp; 6 Morganella morgani. A total of 1863 AST results were produced and compared with BD Phoenix ${ }^{\mathrm{Ts}}$.

\section{Alfred 60 AST agreement for gram-negative bacteria} Overall categorical agreement for Alfred 60 AST was 95\% (1772/1863) for Gram-negative isolates (55 major errors, 31 very major errors and 5 minor errors). The highest agreement was observed with meropenem 97\% (288/297); ampicillin 96\% (290/301); ciprofloxacin 95\% (283/297); ceftriaxone $96 \%$ (75/78) and piperacillin- tazobactam 95\% (280/295) Lower agreement was seen for amikacin 93\% (277/298) and gentamicin 94\% (279/ 297). Out of a total of 55 major errors, $65 \%(36 / 55)$ involved E.coli against amikacin 13; gentamicin 8; piperacillin-tazobactam 6; meropenem 3; ampicillin 4; ciprofloxacin 2 and 20\% (11/55) involved Klebsiella spp. against ciprofloxacin 3; meropenem 3; amikacin 2; piperacillin/tazobactam 2 . Very major errors were seen with ciprofloxacin 6; ampicillin 7; gentamicin 7; piperacillin/ tazobactam 5; meropenem 3; ceftriaxone 2 and amikacin 1. Tables 1 and 2.

Table 1 AST results of the Alfred 60AST ${ }^{\mathrm{TM}}$ system compared to BD Phoenix ${ }^{\mathrm{TM}}$ system for each antibiotic. Column 2 shows the total number of susceptibility tests done with both methods; columns 3 to 6 the total number of tests for which there was agreement within each category and in total; columns 8 to 10 the number of discrepancies; $S=$ susceptible $=$ intermediate, $R=$ resistant, $C A=$ Categorical agreement, $95 \% \mathrm{Cl}=95 \%$ confidence interval

\begin{tabular}{|c|c|c|c|c|c|c|c|c|c|}
\hline \multirow[b]{2}{*}{ Antimicrobial agent } & \multirow[b]{2}{*}{ No. of AST results } & \multicolumn{5}{|c|}{ No. of category agreements } & \multicolumn{3}{|c|}{ No. of discrepancies } \\
\hline & & $S$ & $\mathrm{R}$ & I & CA Total & CA \% $(95 \% \mathrm{Cl})$ & Minor & Major & Very major \\
\hline \multicolumn{10}{|l|}{ Gram-negative antimicrobials } \\
\hline Ampicillin & 301 & 88 & 202 & 0 & 290 & 96 (94-98) & 0 & 4 & 7 \\
\hline Amikacin & 298 & 273 & 2 & 2 & 277 & 93 (89-96) & 3 & 17 & 1 \\
\hline Ciprofloxacin & 297 & 222 & 61 & 0 & 283 & $95(92-97)$ & 1 & 7 & 6 \\
\hline Ceftriaxone & 78 & 64 & 11 & 0 & 75 & 96 (89-99) & 0 & 1 & 2 \\
\hline Gentamicin & 297 & 240 & 38 & 1 & 279 & $94(91-96)$ & 1 & 10 & 7 \\
\hline Piperacillin/Tazobactam & 295 & 263 & 16 & 1 & 280 & $95(92-97)$ & 0 & 10 & 5 \\
\hline Meropenem & 297 & 284 & 4 & 0 & 288 & 97 (94-99) & 0 & 6 & 3 \\
\hline Overall agreement & 1863 & 1434 & 334 & 4 & 1772 & 95 (94-96) & $5(0.3 \%)$ & $55(3 \%)$ & $31(2 \%)$ \\
\hline \multicolumn{10}{|l|}{ Gram-positive antimicrobials } \\
\hline Cefoxitin & 75 & 34 & 35 & 0 & 69 & $92(83-97)$ & 0 & 4 & 2 \\
\hline Cindamycin & 75 & 41 & 17 & 2 & 60 & $80(69-88)$ & 1 & 9 & 5 \\
\hline Teicoplanin & 86 & 79 & 0 & 1 & 79 & $92(84-97)$ & 1 & 6 & 0 \\
\hline Vancomycin & 86 & 76 & 1 & 0 & 77 & $90(81-95)$ & 0 & 9 & 0 \\
\hline Ampicillin & 11 & 9 & 1 & 0 & 10 & $90(59-100)$ & 0 & 1 & 0 \\
\hline Overall agreement & 333 & 239 & 54 & 3 & 295 & 89 (85-92) & $2(0.6 \%)$ & 29 (9\%) & $7(2 \%)$ \\
\hline
\end{tabular}


Table 2 AST results of the Alfred 60 ASTTM $^{T M}$ stem compared to BD Phoenix ${ }^{\mathrm{TM}}$ system for 301 Gram-negative and 86 Gram-positive organisms included in the study. Column 2 refers to the total number of susceptibility tests done with both methods; colum 3 refers to the number of tests for which there was agreement between both tests. $\mathrm{S}=$ sensitivie, I= intermediate, $\mathrm{R}=\mathrm{resistant}, \mathrm{CA}=$ Categorical agreement, $95 \% \mathrm{Cl}=95 \%$ confidence interval. CoNS - Coagulase negative staphylococcus, 95\% Cl- 95\% confidence interval

\begin{tabular}{|c|c|c|c|c|c|c|c|}
\hline & \multirow[b]{2}{*}{ No. of Isolates } & \multirow[b]{2}{*}{ No. of AST results } & \multirow[b]{2}{*}{ No. of CA } & \multirow[b]{2}{*}{ CA \% $(95 \%$ Cl) } & \multicolumn{3}{|c|}{ No. of discrepancies } \\
\hline & & & & & Very major & Major & Minor \\
\hline \multicolumn{8}{|l|}{ Gram-negative organisms } \\
\hline E.coli & 209 & 1291 & 1242 & $96(95-97)$ & 9 & 36 & 4 \\
\hline Klebsiella spp & 57 & 351 & 330 & $94(91-96)$ & 10 & 11 & 1 \\
\hline P.mirabillis & 12 & 79 & 69 & $87(78-84)$ & 6 & 3 & 0 \\
\hline P.aeruginosa & 10 & 61 & 58 & $95(86-91)$ & 0 & 3 & 0 \\
\hline E.cloacae & 7 & 43 & 37 & 86 (72-95) & 0 & 6 & 0 \\
\hline M.morgani & 6 & 38 & 36 & 95 (82-99) & 0 & 2 & 0 \\
\hline Overall Gram-negative & 301 & 1863 & 1772 & 95 (94-96) & 31 & 55 & 5 \\
\hline \multicolumn{8}{|l|}{ Gram-positive organisms } \\
\hline S.epidermidis & 26 & 104 & 98 & $94(88-98)$ & 3 & 2 & 1 \\
\hline S.aureus & 21 & 84 & 66 & $79(68-87)$ & 1 & 17 & 0 \\
\hline S.hominis & 15 & 60 & 54 & 90 (79-96) & 2 & 4 & 0 \\
\hline CoNS other & 13 & 52 & 48 & $92(81-97)$ & 1 & 3 & 0 \\
\hline E.faecalis & 10 & 30 & 27 & 93 (73-98) & 0 & 3 & 0 \\
\hline E.faecium & 1 & 3 & 2 & $67(10-100)$ & 0 & 0 & 1 \\
\hline Overall Gram-positive & 86 & 333 & 295 & 89 (85-92) & 7 & 29 & 2 \\
\hline
\end{tabular}

\section{Alfred 60 AST agreement for gram-positive bacteria}

A total of 86 valid Gram-positive organisms were analysed giving a total of 333 Alfred 60 AST results. The overall categorical agreement for Alfred 60 AST was $89 \%$ (295/333) for Gram-positive samples. The highest agreement was observed with cefoxitin 92\% (69/75) and teicoplanin 92\% (79/86) whilst clindamycin and vancomycin showed the lowest one 80\% (60/75) and 90\% (77/ 86) respectively. There were 29 major errors, 7 very major errors and 2 minor errors. Seventeen of the major errors (68\%) involved S. aureus tested clindamycin 6, teicoplanin 6 and vancomycin 5 . The remaining 12 major errors involved CoNS and Enterococci Spp against vancomycin and cefoxitin. Five of the very major errors involved CoNS tested against clindamycin and two involved S. aureus and Staphylococcus epidermidis tested against cefoxitin. Tables 1 and 2 .

\section{Time to results}

The median time to susceptibility results from flagging positive blood cultures was $6.3 \mathrm{~h}(\mathrm{IQR}=5.25-8.4)$ for $\mathrm{Al}$ fred 60 AST system compared to the $20 \mathrm{~h}(\mathrm{IQR}=16-24)$ for BD Phoenix ${ }^{\mathrm{TM}}$. As a result, a reduction in the turnaround time by $13 \mathrm{~h}$ when compared to BD Phoenix ${ }^{\text {тм }}$ was observed.

In terms of "hands-on-time", in our experience, an average time of $20 \mathrm{~min}$ were required to load the samples on the Alfred rapid system, comparable to 15 min for BD Phoenix ${ }^{\mathrm{TM}}$ system. Table 3.

\section{Discussion}

This study shows the performance of Alfred $60 \mathrm{AST}^{\mathrm{rm}}$ system, which significantly reduced the time to antimicrobial susceptibility results. The overall categorical agreement for Alfred 60 AST system was $96 \%$ for Gramnegative and $90 \%$ for Gram-positive organisms. These results are broadly comparable to an earlier smaller study reported by Barnini et al [12], which compared the Alfred $60 \mathrm{AST}^{\mathrm{TM}}$ with VITEK-2 ${ }^{\mathrm{TM}}$, and reported a categorical agreement of $91 \%$ for enterobacteraciae and $94 \%$ for staphylococci. Although similar, differences in the performances may be due to the choice of antibiotics tested, Barnini et al tested ceftazidime and gentamicin, but did not test amoxicillin and ciprofloxacin.

Compared to the BD Phoenix, the overall categorical agreement for Alfred 60 AST system was 96\% for Gramnegative and $90 \%$ for Gram-positive organisms. Previous studies report the accuracy of the BD Phoenix ${ }^{\mathrm{rm}}$ system for susceptibility results compared against agar dilution and manual disc diffusion, showing categorical agreement of 97.5\% [13] and 95\% (Donay, 2004) respectively.

Alfred $60 \mathrm{AST}^{\mathrm{TM}}$ system achieved a performance of over 95\% CA compared to the BD Phoenix ${ }^{\text {Ts }}$ for all antibiotics except amikacin and gentamicin. However, this new 
Table 3 Turnaround time for results from blood culture positivity: Median Time (in hours) and Interquartile range from positive blood culture to results of Alfred vs BD Phoenix ${ }^{\mathrm{TM}}$. Time in hours from positive blood culture to susceptibility results

\begin{tabular}{llll}
\hline & Alfred 60 AST & BD Phoenix $^{\text {TM }}$ system & Alfred $^{\text {TM }}$ vs BD Phoenix \\
\hline Time to results from positive blood culture (h) & $6.3 \mathrm{~h}(\mathrm{IQR}, 5.25-8.25)$ & $20 \mathrm{~h}(\mathrm{IQR}, 16-24)$ & $\mathrm{p}<0.01$ \\
Technician "hands-on" time ( $\mathrm{min})$ & $20 \mathrm{~min}(\mathrm{IQR}, 10-30)$ & $15 \mathrm{~min}(\mathrm{IQR}, 10-20)$ & \\
Technique Characteristics & Direct culture bottle & Agar plate subculture (4 h) & \\
\hline
\end{tabular}

system showed slightly lower performance for Grampositive isolates and for glycopeptides and clindamycin.

In our study, the average hands-on time per specimen was not different from routine testing, however the time to deliver susceptibility results has been significantly reduced compared to routine standard automated system (BD Phoenix $\left.x^{\mathrm{Tr}}\right)$. The times to AST results were similar to the times noted in a previous study using the Alfred 60 $\mathrm{AST}^{\mathrm{mm}}$ system and other the Accelerate Pheno ${ }^{\mathrm{Tm}}$ system (Accelerate Diagnostics, USA) [12] [15] [16] [17]. Given high categorical agreement between the BD Phoenix ${ }^{\text {tax }}$ and Alfred $60 \mathrm{AST}^{\mathrm{Tm}}$ and the $16 \mathrm{~h}$ reduction in time to results there are compelling clinical benefits of the Alfred AST $60^{\mathrm{Tm}}$.

The Accelerate Pheno ${ }^{\text {Tw }}$ system is an alternative rapid phenotypic testing system. In studies using similar AST comparator techniques, an agreement of 93.3\% [18], 94.9\% [19] and $96.4 \%$ respectively [15] has been reported. Showing very similar diagnostic performance to the Alfred AST $60^{\mathrm{m}}$. The median time to AST results from positive culture bottle to AST result for the Accelerate Pheno ${ }^{\text {Tx }}$ system is reported as 10.7 [8.6-12.8] hours [15]. It should be noted that an advantage of the Accelerate Pheno ${ }^{\text {Tx }}$ system is that it identifies the species of the isolate in addition to the sensitivity pattern. For this reason, we needed to perform a rapid identification with a MALDI-TOF MS at the same time as the Alfred 60 $\mathrm{AST}^{\mathrm{m}}$.

A specific advantage of the Alfred 60 AST $^{\mathrm{Tx}}$ system versus other rapid phenotypic susceptibility methods is the capacity to accommodate between 7 and 9 isolates per run, the number of samples may be varied according to the customized antibiotic panel chosen by the user and a satellite module provides extra capacity to handle up to a total of 20 isolates per day. Furthermore, new samples can be loaded during an ongoing run without interfering previous loaded isolates.

The cost of performing rapid phenotypic sensitivity testing for some systems has been regarded as high and seen as a barrier to introducing these tests. We estimate the additional reagent costs for the Alfred $60 \mathrm{AST}^{\mathrm{max}}$ of $€ 2-4$ per antibiotic tested [20] or €30-40 per isolate, with relatively minimal laboratory hands on time. This cost is considerably lower than other rapid testing systems and we believe could be justified by the clinical benefits.
One of the limitations of the system is it will not perform antimicrobial susceptibility testing on polymicrobial samples, although Gram-staining before using the Alfred $60 \mathrm{AST}^{\mathrm{max}}$ meant polymicrobial samples were relatively uncommon in these samples. Another possible drawback is the absence of MIC values, which are not provided on the susceptibility report, that may occasionally be relevant for specific therapeutic decisions [21], however most clinical decisions can be made simply with an $\mathrm{S} / \mathrm{I} / \mathrm{R}$ result. Another limitation lies on the inability of performing susceptibility testing of Acinetobacter baumannii or Stenotrophomona maltophila which are emerging as a challenging to treat nosocomial pathogens [22]. Furthermore, there are inconsistencies with the concentration of cefoxitin in the Alfred $60 \mathrm{AST}^{\mathrm{ru}}$ panel. The EUCAST breakpoint for cefoxitin is $4 \mathrm{mg} / \mathrm{L}$ for $S$. aureus and S. lugdunensis and $8 \mu \mathrm{g} / \mathrm{L}$ for Staphylococcus saprophyticus, whilst Alfred 60AST ${ }^{\mathrm{mix}}$ breakpoint for all coagulase negative Staphylococci is $8 \mu \mathrm{g} / \mathrm{L}$.

Limitations of the study were that although broth microdilution is considered the "gold standard" technique for antimicrobial susceptibility evaluation, this is rarely routinely available in practice in clinical laboratories. The BD Phoenix ${ }^{\mathrm{mat}}$ system is a well validated automated AST system [13]. Similar methodologies of comparing rapid diagnostic tests with automated AST systems in place of broth or agar dilution has been used in majority of published studies [19] [16] [15] [17] [18] [13].

We note the relatively small proportion of multiresistant microorganisms in our particular setting and the fact that more than half of the susceptibility testing for Gram-negative isolates were performed for E.coli blood stream infections. Furthermore, a smaller number of Gram-positive organisms were tested compared to the Gram-negative organisms.

Even though there is clinical utility in rapid antimicrobial sensitivity results we would still recommend isolates have further extensive testing with another system, such as the BD Phoenix ${ }^{\mathrm{Tu}}$ or a VITEK ${ }^{\mathrm{Tn}} 2$, to give a complete sensitivity pattern to a wider range of antibiotics and give results for minimum inhibitory concentrations. Since, BD phoenix shows more accuracy specially for gram positive bacteria.

The implementation of the Alfred $60 \mathrm{AST}^{\mathrm{Tw}}$ system in the laboratory routine workflow, will provide rapid 
susceptibility results, that effectively communicated to clinicians might result in a prompt change to appropriate antimicrobial treatment, antimicrobial resistance reduction and improved infection control [23] [24] [25].

\section{Conclusions}

In conclusion, Alfred $60 \mathrm{AST}^{\mathrm{mm}}$ system has demonstrated an improvement in the turn-around time of antimicrobial phenotypic susceptibility results in Gram-negative and Gram-positive blood stream infections in comparison with automated AST techniques and conventional AST testing.

Whilst the system provided a notably good performance and high accuracy for Gram-negative bacteria, it still needs improvements in Gram-positive AST results and is less dependable for yeast detection [20].

Prospective studies are needed to further analyse the impact and effects of the rapid diagnostic techniques among bacteraemia patients and consequences of the introduction of new available rapid automated systems on antibiotic prescription against BSI and on clinical patient pathways.

\section{Methods}

\section{Study setting}

A prospective evaluation was conducted directly from blood cultures, between January and May 2018 in the microbiology laboratory of South West London Pathology in London. The diagnostic laboratory processes approximately 3500 positive blood cultures per year. All blood culture samples were initially incubated on the BD BacTec FX400 (Becton Dickinson, USA). Blood culture bottles, including adult and paediatric samples, that flagged positive were then tested in parallel for antimicrobial susceptibility by Alfred 60 AST and by routine testing on the BD Phoenix ${ }^{\text {tm }}$, after Gram staining performance to determine a Gram-positive or Gramnegative antibiotic panel required and exclude the polymicrobial samples.

Bacterial species were identified by Bruker Biotyper matrix assisted laser desorption ionization-time of flight mass spectrometry (MALDI-TOF MS, Germany), this technique was performed, as per routine laboratory practice, from isolated colonies obtained following $4 \mathrm{~h}$ agar plate subculture, in order to fully interpret the results of the Alfred 60AST with species ID. This meant the species of the samples were identified before the Alfred 60AST result was available. (Fig. 2).

\section{Alfred 60 AST system}

The Alfred 60 ST was performed according to the manufacturer's instructions on blood culture bottle that had flagged positive on the BD BacTec FX system, this

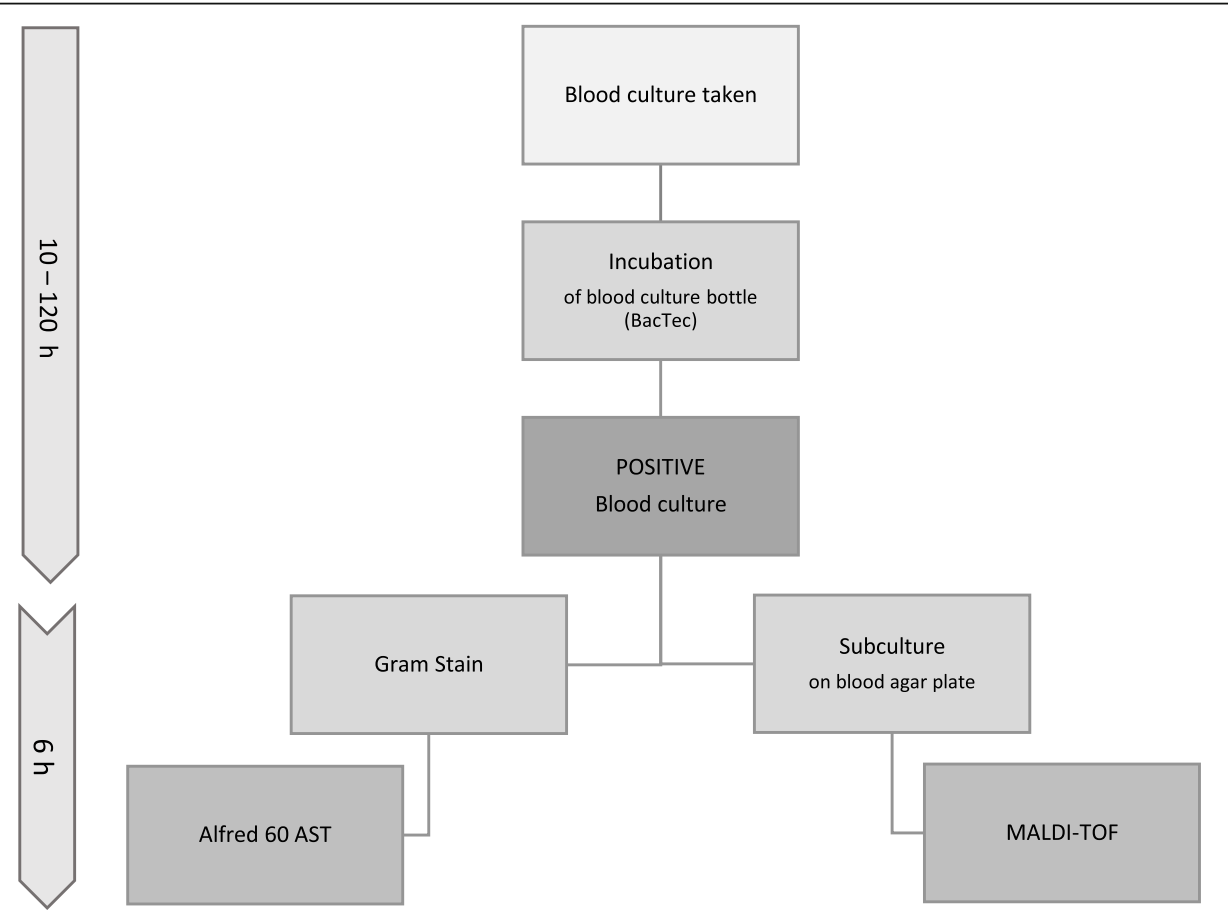

Fig. 2 Flowchart showing laboratory workflow followed along the rapid susceptibility system (Alfred 60 AST) evaluation. Once a blood culture bottle flagged positive following incubation, Gram-stain was performed in order to distinguish between Gram-positive and Gram-negative microorganism and exclude the polymicrobial ones, which will allow to select the appropriate antibiotic panel and a direct blood sample from the bottle was loaded onto the rapid system. In parallel, the identification results were obtained by MALDI-TOF, performed from isolated colonies obtained by $4 \mathrm{~h}$ agar plate subculture. The mean total time from positive blood culture to identification and susceptibility results was $6 \mathrm{~h}$ (approx) 


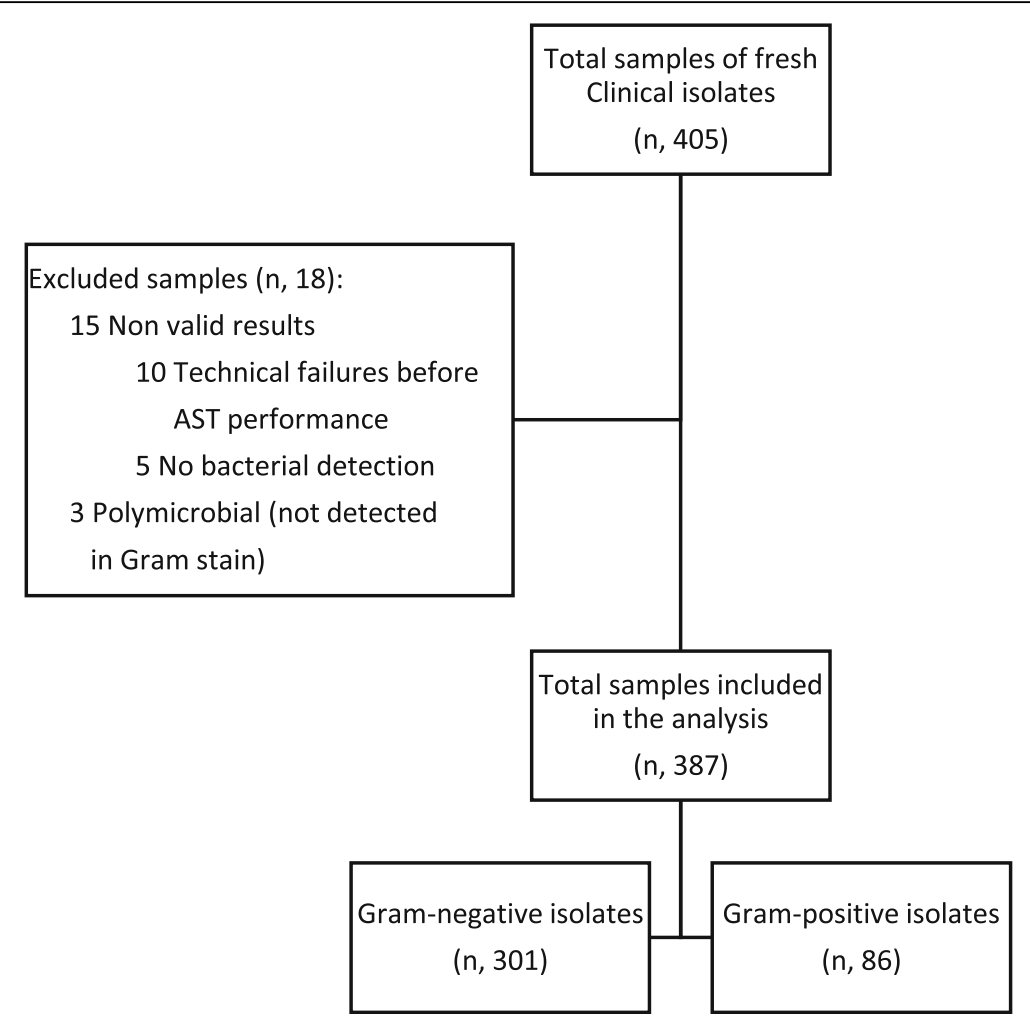

Fig. 3 Flowchart of study population. A total of 387 (301 Gram-negative and 86 Gram-positive) valid monomicrobial clinical isolates after 18 excluded samples. Excluded samples comprised 3 polymicrobial and 15 non valid results included 10 system technical failure and 5 no bacterial detection and subsequently no AST performance

including running weekly controls with Pseudomonas aeruginosa 27,853; E.coli 25,922; E.coli 35,218; S.aureus 29,213; E.faecalis 29,212; E. faecalis $51,299.30 \mu \mathrm{L}$ of the sample was inoculated into an enrichment broth and loaded onto the instrument. Each vial of antibiotic contains $45 \mathrm{mg}$ of a preparation of lyophilized antibiotic that required to be dissolved in $2 \mathrm{~mL}$ of regenerating solution, as per manufacturer's specifications. Some antibiotics, including amikacin, piperacillin-tazobactam and meropenem, required different specific antibiotic vials for testing Enterobacteriaceae and Pseudomonas Spp. As the identity of organism is unknown when the Alfred 60 AST system was set up, a combined panel of antibiotics with both concentrations was used. When the identity of the organism was known the appropriate antibiotic result was reported.

\section{Antimicrobial agents}

The following antibiotic panel was performed on Alfred $60 \mathrm{AST}^{\mathrm{Tm}}$ for all Gram-negative organisms (Enterobacteriaceae and Pseudomonas spp):: ampicillin, amikacin, ciprofloxacin, gentamicin, meropenem, and piperacillintazobactam. Ceftriaxone was included on the Enterobacteriaceae panel later in March 2018 and tested in 78 isolates. For Gram-positive organisms the antibiotic panel included: ampicillin, cefoxitin, clindamycin, teicoplanin and vancomycin. Purity plates were set up both on CLED agar and blood agar plates and examined the next day to confirm that the inoculum was not mixed.

\section{Comparator AST technique}

The BD Phoenix ${ }^{\mathrm{ma}}$ automated susceptibility testing system CE marked IVD (BD Diagnostics, Sparks, MD, USA) (software version $5.02 \mathrm{H} / 4.11 \mathrm{~B}$ ) was used as the routine laboratory method according to the manufacturer instructions, including running weekly controls with Pseudomonas aeruginosa 27,853; E.coli 25,922; E.coli 35,218; S.aureus 29,213; E.faecalis 29,212; E. faecalis 51,299 using cartridge PMIC-96 for Gram-positive and NMIC-417 for Gram-negative isolates.

\section{Interpretation and comparison of results}

Isolates were classified as susceptible, intermediate or resistant (S/I/R) according to interpreted reports provided by Alfred 60AST ${ }^{\mathrm{Tm}}$ and BD Phoenix ${ }^{\mathrm{Tm}}$. The final report interpretation of susceptibility results provided by Alfred $60 \mathrm{AST}^{\mathrm{Tn}}$ are based on bacterial growth curve analysis Fig. 1.

Each sample result by Alfred 60 AST system was compared against BD Phoenix ${ }^{\mathrm{min}}$. Categorical agreement (CA) 
was defined as agreement of test results interpreted within the same susceptibility category(S/I/R).

Discordant results were categorized as very major error (VME, reported susceptible on Alfred AST60 ${ }^{\mathrm{mm}}$ when reported resistant Phoenix ${ }^{\mathrm{mi}}$ ), major error (ME, reported resistant when susceptible) or minor error $(\mathrm{mE}$, reported intermediate when susceptible or resistant). The criteria for antimicrobial susceptibility testing proposed by Jorgensen has been used [26] [27].

\section{Time to AST results}

The time to AST was defined as the time between the culture bottle flagging positive on the BacTec and the availability of the AST result by Alfred 60 AST system or BD Phoenix ${ }^{\mathrm{mm}}$. Wilcoxon test was performed for comparison of median AST time analyses, considering $p<$ 0.05 as a significant value.

SPSS (Statistical Package for the Social Sciences, IBM, USA) version 25.0 statistics software was used for all analyses.

\begin{abstract}
Abbreviations
AST: Antimicrobial susceptibility testing; BSI: Blood stream infections; CA: Categorical agreement; CoNS: Coagulase negative Staphylococci; ESBLs: Extended-spectrum beta-lactamases; EUCAST: European Committee on Antimicrobial Susceptibility Testing; MALDI-TOF: Matrix-Assisted Laser Desorption/lonization-Time Of Flight; ME: Major error; mE: minor error; MIC: Minimum inhibitory concentration; S/ I/ R: Susceptible/ Intermediate/ Resistant; UK HRA: United Kingdom Health Research Authority; VME: Very major error
\end{abstract}

\section{Acknowledgments}

We thank the team of laboratory technicians at St. George's University Hospital for their valuable work and technical assistance.

This study forms a part of the MD (Res) thesis of Dr. Vanesa Anton-Vazquez.

\section{Data privacy and confidentiality}

Access to patient records and data was granted to an approved clinical research fellow. Patient data was anonymised and securely protected to ensure patient confidentiality.

\section{Consent to publication}

Not applicable.

\section{Author's contributions}

VAV, SA, CS \& TP designed the study. VAV \& SA ran the assays. Writing and analysis performed by VAV, SA, CS \& TP.

All authors (VAV, SA, CS \& TP) have read and approved the manuscript.

\section{Funding}

The study was funded by the National Institute for Health Research (NIHR) Collaboration for Leadership in Applied Health Research and Care (CLAHRC) South London, UK.

The funding body had no role in study design, data collection, analysis, interpretation of data, writing the manuscript and decision to publish.

\section{Availability of data and materials}

Raw data used in the study are provided in the form of excel spreadsheets on the SGUL data repository. https://sgul.figshare.com/s/c8746f6e579802 $78 \mathrm{bb} 4 \mathrm{f}$

DOI https://doi.org/10.24376/rd.sgul.9782243

\section{Ethics approval and consent to participate}

This study reports the findings of the laboratory verification of the Alfred 60AST. No personal information was stored in the study database. All samples taken from patients were collected as part of standard care and all tests ran on them were also part of the standard care procedure, as is standard practice for a laboratory verification for the introduction of new equipment in the laboratory, specific approval was not necessary under UK HRA decision tools.

\section{Competing interests}

Test reagents were provided by Alifax, Italy, for the study period. Alifax had no role in study design, data collection, data analysis or study submission for publication.

\section{Author details}

${ }^{1}$ St. George's University of London. Institute for Infection and Immunity, London SW17 ORE, UK. ²Department of Medical Microbiology, Southwest London Pathology, St. George's Hospital Blackshaw Road, London SW17 OQT, UK. ${ }^{3}$ Infection Care Group, St George's University Hospitals NHS Foundation Trust, Blackshaw Road, London SW17 0QT, UK.

Received: 25 May 2019 Accepted: 18 November 2019

Published online: 29 November 2019

\section{References}

1. Adhikari NK, Fowler RA, Bhagwanjee S, Rubenfeld GD. Critical care and the global burden of critical illness in adults. Lancet. 2010;376:1339-46. https:// doi.org/10.1016/S0140-6736(10)60446-1.

2. Anderson DJ, Moehring RW, Sloane R, Schmader KE, Weber DJ, Fowler VG, et al. Bloodstream Infections in Community Hospitals in the 21 st Century: A Multicenter Cohort Study. PloS One. 2014;9(3):1-10. https://doi.org/10.1371/ journal.pone.0091713.

3. Goto M, Al-Hasan MN. Overall burden of bloodstream infection and nosocomial bloodstream infection in North America and Europe. Clin Microbiol Infect. 2013;19:501-9.

4. Maurer FP, Christner M, Hentschke M, Rohde H. Advances in rapid identification and susceptibility testing of bacteria in the clinical microbiology laboratory: implications for patient care and antimicrobial stewardship programs. Infect Dis Rep. 2017;9(1):18-27.

5. Meda M, Clayton J, Varghese R, Rangaiah J, Grundy C, Dashti F, et al. What are the critical steps in processing blood cultures? A prospective audit evaluating current practice of reporting blood cultures in a centralised laboratory serving secondary care hospitals. J Clin Pathol. 2017;70(4):361.

6. Romero-Gómez MP, Gómez-Gil R, Paño-Pardo JR, Mingorance J. Identification and susceptibility testing of microorganism by direct inoculation from positive blood culture bottles by combining MALDI-TOF and Vitek-2 compact is rapid and effective. J Inf Secur. 2012;65:513-20. https://doi.org/10.1016/j.jinf.2012.08.013.

7. Buehler SS, Madison B, Snyder SR, et al. Effectiveness of practices to increase timeliness of providing targeted therapy for inpatients with bloodstream infections: a laboratory medicine best practices systematic review and meta-analysis. Clin Microbiol Rev. 2016; 29:59-103.

8. Caliendo AM, Gilbert DN, Ginocchio CC, et al. Better tests, better care: improved diagnostics for infectious diseases. Clin Infect Dis. 2013;57(Suppl 3):S139.

9. Warhurst G, Dunn G, Chadwick P, Blackwood B, McAuley D, Perkins GD, et al. Rapid detection of health-care-associated bloodstream infection in critical care using multipathogen real-time polymerase chain reaction technology: a diagnostic accuracy study and systematic review. Health Technol Assess. 2015;19(35).

10. Timbrook TT, Morton JB, McConeghy KW, et al. The effect of molecular rapid diagnostic testing on clinical outcomes in bloodstream infections: a systematic review and meta-analysis. Clin Infect Dis. 2017;64:15-23.

11. Kerremans JJ, Verboom P, Stijnen T, et al. Rapid identification and antimicrobial susceptibility testing reduce antibiotic use and accelerate pathogen-directed antibiotic use. J Antimicrob Chemother. 2008;61:428-35.

12. Barnini S, Brucculeri V, Morici P, Ghelardi E, Florio W, Lupetti A. A new rapid method for direct antimicrobial susceptibility testing of bacteria from positive blood cultures. BMC Microbiol. 2016;16(1):185.

13. Carroll KC, Glanz BD, Borek AP, et al. Evaluation of the BD Phoenix automated microbiology system for identification and antimicrobial susceptibility testing of Enterobacteriaceae. J Clin Microbiol. 2006;44:3506-9.

14. Donay JL1, Mathieu D, Fernandes P, Prégermain C, Bruel P, Wargnier A, et al. Evaluation of the automated phoenix system for potential routine use in the clinical microbiology laboratory. J Clin Microbiol. 2004;42(4):1542-6. 
15. Descours G, Desmurs L, Hoang TLT, et al. Evaluation of the accelerate Pheno system for rapid identification and antimicrobial susceptibility testing of gram-negative bacteria in bloodstream infections. Eur J Clin Microbiol Infect Dis. 2018:37:1573-83.

16. Lutgring JD, Bittencourt C, McElvania TE, Cavuoti D, Hollaway R, Burd EM. Evaluation of the Accelerate Pheno System: Results from Two Academic Medical Centers. J Clin Microbiol. 2018;56(4). https://doi.org/10.1128/JCM. 01672-17.

17. Brazelton de Cardenas JN, Su Y, Rodriguez A, Hewitt C, Tang L, Garner CD, Hayden RT. Evaluation of rapid phenotypic identification and antimicrobial susceptibility testing in a pediatric oncology center. Diagn Microbiol Infect Dis. 2017;89:52-7.

18. Marschal M, Bachmaier J, Autenrieth I, Oberhettinger P, Willmann M, Peter S. Evaluation of the accelerate Pheno system for fast identification and antimicrobial susceptibility testing from positive blood cultures in bloodstream infections caused by gram-negative pathogens. J Clin Microbiol. 2017;55(7):2116-26.

19. Pantel A, Monier J, Lavigne JP. Performance of the accelerate Pheno TM system for identification and antimicrobial susceptibility testing of a panel of multidrug-resistant gram-negative bacilli directly from positive blood cultures. J Antimicrob Chemother. 2018;73(6):1546-52.

20. Lahanas S, Stathopoulos G, Chan RC, van Hal SJ. Evaluation of the Alfred 60/ AST device as a screening test for urinary tract infections. J Clin Microbiol. 2013;51(10):3406-8.

21. Roberts JA, Paul SK, Akova M. Defining antibiotic levels in intensive care unit patients: are current beta-lactam antibiotic doses sufficient for critically ill patients? Clin Infect Dis. 2014;58(8):1072-83.

22. Coelho J, Woodford N, Turton J, Livemore DM. Multiresistant Acinetobacter in the UK: how big a threat? J Hosp infect. 2004;58:167-9.

23. Lee C-C, Lee C-H, Hong M-Y, Tang H-J, Ko W-C. Timing of appropriate empirical antimicrobial administration and outcome of adults with community-onset bacteremia. Crit Care. 2017;21(1):119.

24. Nimmich EB, Bookstaver PB, Kohn J, Justo JA, Hammer KL, Albrecht $\mathrm{H}$, et al. Development of institutional guidelines for management of Gram-negative bloodstream infections: Incorporating local evidence. Hosp Pharm. 2017; 52(10):691-7. https://doi.org/10.1177/0018578717720506.

25. Cain SE, Kohn J, Bookstaver PB, Albrecht H, Al-Hasan MN. Stratification of the impact of inappropriate empirical antimicrobial therapy for gramnegative bloodstream infections by predicted prognosis. Antimicrob Agents Chemother. 2015;59:245-50.

26. Jorgensen $\mathrm{KH}$. Selection criteria for an antimicrobial susceptibility testing system. J Clin Microbiol. 1993;31:2841-4.

27. Jorgensen $J H$, Ferraro MJ. Antimicrobial susceptibility testing: a review of general principles and contemporary practices. Clin Infect Dis. 2009 Dec 1; 49(11):1749-55. https://doi.org/10.1086/647952.

28. Buehler SS, Madison B, Snyder SR, et al. Effectiveness of practices to increase timeliness of providing targeted therapy for inpatients with bloodstream infections: a laboratory medicine best practices systematic review and meta-analysis. Clin Microbiol Rev. 2016;29:59-103.

29. European Committee for Antimicrobial Susceptibility Testing of the European Society of Clinical Microbiology and Infectious Diseases EUCAST determination of antimicrobial susceptibility test breakpoints. 2018.

\section{Publisher's Note}

Springer Nature remains neutral with regard to jurisdictional claims in published maps and institutional affiliations. 